\title{
La conductivité électrique des eaux naturelles ses variations et leurs applications
}

\section{The electrical conductivity of natural water. Its variations and applications.}

\section{J. BONNIN}

INGÉNIEUR AU SERVICE DES ÉTUDES HYDRAULIQUES D'E.D.F.
PAR

$\mathrm{ET}$
Influence des sels dissous sur la conductivité des eaux naturelles.

Augmentation de la conductivité vers l'aval. Diminution de la conductivité lorsque le débit augmente.

Vitesse de dissolution des sels.

Forme des lois de variations de la conductivité en fonction de l'abscisse et du débit.

Application de ces lois : complexité d'un système hydrographique.

Application $\dot{a}$ un cours d'eau uniforme sans affluent. Vérification de la loï d'abscisse.

Remarque sur les valeurs obtenues pour la saturation. Fausse saturation. Vérifications expérimentales.

Détermination expérimentale de la loi de débit. Application de la loi de débit. Courbes de tarage.

Extrapolation de la loi d'abscisse. Loctlisation des sources. Application.

Conclusion.

A la suite des travaux présentés au Comité technique le 20 novembre 1953 par M. Ravier, nous avons pensé lier plus intimement les variations spatiales et temporelles de la résistivité en les rattachant à la dissolution lente des roches par les eaux et donner à leurs lois une forme plus rigoureuse.
The influence of dissolved salts on the conductivité of natural water.

Increase in conductivity towards the downstream.

Decrease in conductivity with increase in flow. speed of dissolution of the salts.

Form of the laws of conductivity variation in terms of the abscissa and the flow.

Application of these laws. Complexity of an hydrographical system.

Application to a water course without a tributary. Verification of the abscissa law.

Remark on the values obtained for saturation. False saturation. Experimental verification..

Experimental determination of the discharge law.

Application of the flow law. Calibration curve. Extrapolation of the abscissa law. Location of sources. Applications.

Conclusions.

Bien que nos études soient loin d'être terminées, nous avons obtenu des résultats qui nous semblent permettre de serrer de plus près la vérité et d'ouvrir de nouvelles perspectives à cette méthode d'investigation qui s'est déjà révélée féconde en hydrologie.

\section{INFLUENCE DES SELS DISSOUS SUR LA CONDUCTIVITÉ DES EAUX NATURELLES}

La communication de M. Ravier mettait en évidence l'influence de la nature des roches soumises à l'érosion torrentielle. L'indice minéralogique de solubilité, qui correspond à la conductivité maximum des eaux (conductivité obte- nue théoriquement pour un débit nul), est un caractère spécifique de la géologie et de la pétrographie du bassin versant. C'est le produit par $10^{5}$ de la conductivité maximum exprimée en mhos/cm. Dans tout ce qui suit, nous serons 
d'ailleurs amenés à raisonner sur la conductivité $\lambda$ plutôt que sur son inverse la résistivité et, pour des raisons d'ordre de grandeur, nous exprimerons $\lambda$ en micromhos, c'est-à-dire que les

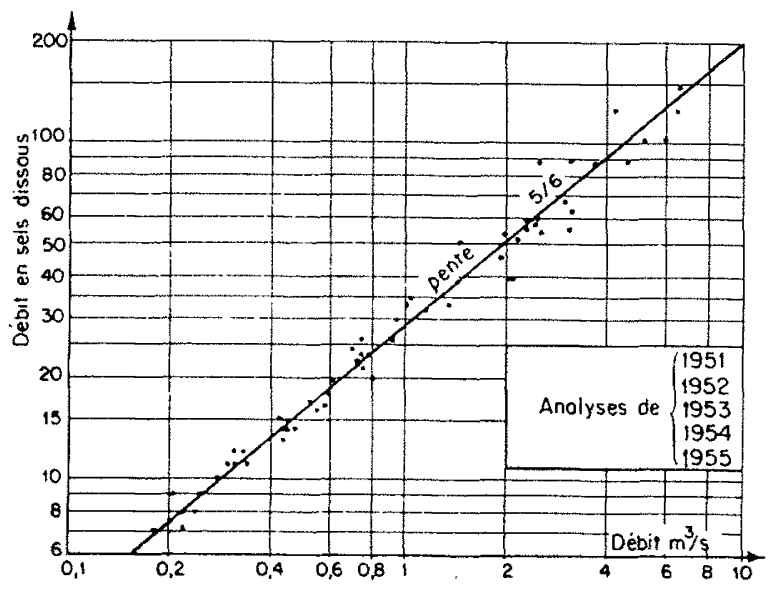

Frg. 1. - Gave d'Arrens. Station de Suyen.

valeurs numériques seront dix fois supérieures à celles de l'indice minéralogique de solubilité.

Une étude publiée en 1953 par M. Walton H. Durum $\left({ }^{\star}\right)$ et concernant les corps minéraux dissous dans la rivière Saline, met en évidence la corrélation étroite qui existe entre la conductivité de l'eau de la rivière et sa teneur en différents sels.

Nous avons essayé de relier directement au débit la quantité de matière dissoute, de manière à démontrer formellement son influence.

Plus d'une soixantaine d'analyses ont été effectuées au Laboratoire du Service Hydrologie à Argelès sur les eaux du gave d'Arrens, prélevées à la station du lac de Suyen, entre 1951 et 1956. Elles consistaient à doser l'extrait sec à $110^{\circ} \mathrm{C}$. La figure 1 résume les résultats obtenus, en donnant le débit du torrent en sels dissous en fonction de son débit liquide.

On constate que lorsque le débit $\mathrm{Q}$ augmente, le débit salin augmente lui aussi, sensiblement comme la puissance $5 / 6$ du précédent. En conséquence, la concentration diminue comme $\mathrm{Q}^{-1 / 6}$; nous avons observẻ que, corrélativement, la conductivité diminue.

D'autre part, l'augmentation de la conductivité, lorsque l'on examine des échantillons pris de plus en plus vers l'aval du cours d'eau, s'explique par le fait que les eaux se chargent de plus en plus en matières solides en solution et, par suite, se rapprochent de leur saturation.

\section{VITESSE DE DISSOLUTION DES SELS}

Pour mieux analyser ce phénomène, nous sommes amenés à formuler maintenant une hypothèse concernant la vitesse de dissolution dans l'eau de différents sels contenus dans les roches. Cette vitesse de dissolution dépend de nombreux paramètres : vitesse et composition chimique de l'eau, nature et granulométrie des roches, etc.; il est particulièrement important de la lier à la teneur des eaux en sels.

Il nous paraît raisonnable d'admettre, sous réserve de vérification, que, toutes choses égales d'ailleurs, la vitesse de dissolution est proportionnelle à l'écart entre la concentration actuelle des eaux et la concentration de saturation. Nous pouvons lier immédiatement concentration et conductivité puisqu'elles varient proportionnellement l'une à l'autre tant que la conductivité ne dépasse pas 500 micromhos environ (nous verrons que nous avons rarement atteint plus de 200 à 250 micromhos dans nos observations); ceci nous permet d'écrire l'équation différentielle

(*) WALtos H. DURUM : Relationship of the Mineral constituents in solution to stream flow, Saline river near Russel, Kansas.-Transactions of American Geophysical Union, juin 1953. de la variation de conductivité sous la forme:

$$
\frac{d \lambda}{d t}=\mathrm{K} \frac{f(\mathrm{Q})}{\mathrm{Q}} g(\mathrm{~V})\left(\lambda_{m}-\lambda\right)
$$

Dans cette équation, $\lambda_{m}$ représente la conductivité maximum, c'est-à-dire celle correspondant à la saturation des eaux (il peut s'agir d'une fausse saturation, comme nous le verrons plus loin). Le débit présent au dénominateur montre que la quantité de matière dissoute est diluée dans le débit $Q$.

Pour intégrer cette équation, il est nécessaire de formuler de nouvelles hypothèses.

Si l'on considère un troncon de cours d'eau que nous appellerons " uniforme », c'est-à-dire dans lequel les différents éléments (vitesse de l'eau, débit, périmètre mouillé, nature des roches, granulométrie, etc.) restent invariables, les différents termes de l'équation (1) restent constants à l'exception de $\lambda$ et de $t$; en écrivant que $d x=\mathrm{V} d t$, l'intégration de l'équation (1) donne la forme (2):

$$
\log \frac{\lambda_{m}-\lambda_{0}}{\lambda_{m}-\lambda}=\Phi(Q)\left(x-x_{0}\right)
$$


dans laquelle $\lambda_{0}$ représente la conductivité initiale de l'eau, c'est-à-dire ce qu'elle avait en passant à l'abscisse où elle est entrée dans le troncon considéré.

Le terme $\Phi(Q)$ groupe tous les éléments qui dépendent đu débit et en particulier le terme
1/Q souligné précédemment. On voit que cette hypothèse, déjà assez éloignée de la réalité (cours d'eau « uniforme » sans affluent), nous conduit à une forme encore compliquée dans laquelle le débit intervient d'une façon encore indéterminée.

\section{RECHERCHE D'UNE LOI CONDUCTIVITÉ - DÉBIT}

Pour lever cette indétermination, on peut procéder de trois manières :

- soit essayer des formes simples de la loi $\Phi(\mathrm{Q})$;

- soit chercher la forme de cette loi par des données théoriques;

- soit enfin esayer de l'obtenir par observation ou expérimentation.

a) La première idée que nous avons adoptée consiste à négliger la plupart des facteurs intervenant dans $\Phi(Q)$ et à ne conserver que le terme 1/Q.

Cette hypothèse correspond assez bien au cas d'un cours d'eau à fond affouillable où la dissolution s'opère essentiellement dans les dépôts charriés; en, effet, pour les débits faibles et moyens, c'est-à-dire en fait en l'absence de charriage, les surfaces de contact et la granulométrie des roches ne varient pas; d'autre part, la vitesse de l'eau qui les baigne est elle-même constante, puisqu'il s'agit alors d'un écoulement de filtration pour lequel le gradient longitudinal de $(p+\bar{\omega} h)$ est en moyenne indépendant du débit $Q$.

Nous avons done tenté d'appliquer l'équation (2) sous la forme (3) :

$$
\log \frac{\lambda_{m}-\lambda_{0}}{\lambda_{m}-\lambda}=\frac{\mathrm{K}}{\mathrm{Q}}
$$

et avons constaté qu'elle cadrait bien avec un bon nombre d'observations aux stations de jaugeage; c'est ce que montre en particulier la figure 2, sur laquelle nous avons mis sous cette forme les variations de conductivité en fonction du débit pour! la crue de mars 1951 observée à Geu-Pibeste sur le gave de Pau.

On constate sur cette figure une très faible dispersion des points expérimentaux autour de la droite et une très bonne linéarité qui semblerait apporter une preuve formelle des différentes hypothèses que nous avons faites. En réalité, nous verrons plus loin que cette preuve est moins forte.

b) Nous avons également tenté de mettre la loi $\Phi(Q)$ sous une forme $Q^{n}$ différente de la forme précédente $1 / Q$ en nous appuyant sur les considérations présentées au Comité technique en 1953 par M. Laszloffy. Cette hypothèse serait à retenir lorsque la cause prépondérante de dissolution est l'écoulement à grande vitesse sur le lit. Nous pouvons admettre alors que la vi-

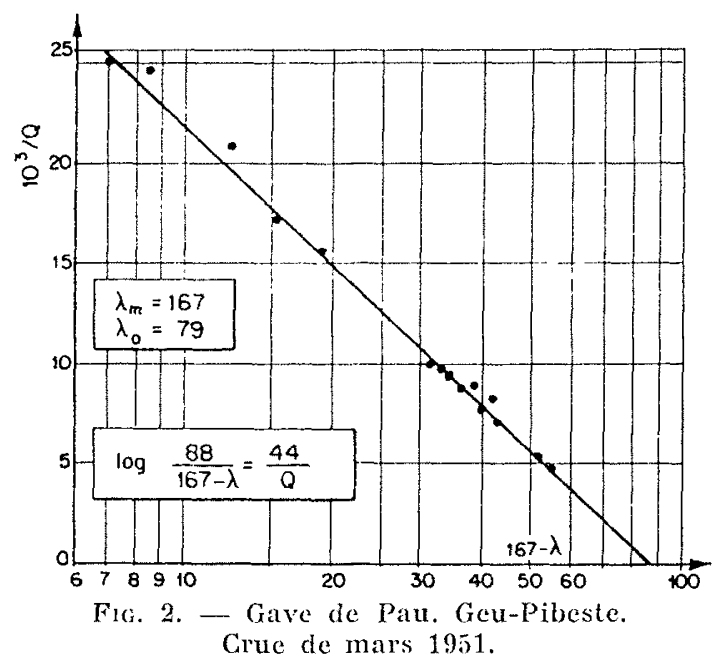

tesse de dissolution varie comme une certaine puissance de la vitesse, celle-ci étant liée par ailleurs, ainsi que le périmètre mouillé, au débit et à la hauteur également par des fonctions monomes.

Nous avons pu mettre la courbe de tarage du gave de Pau à Geu-Pibeste sous une telle forme, comme le montre la figure 3 , qui permet d'écrire:

$$
\mathrm{Q}=\mathrm{K}(\mathrm{H}+0,20 \mathrm{~m})^{1,87}
$$

On peut admettre, pour un cours d'eau comme le gave de $\mathrm{Pau}$, que le périmètre mouillé $\mathrm{L}$ varie comme une puissance du tirant d'eau comprise entre 0 et 0,3 ; d'autre part, M. LAszloffy indique une variation de la vitesse en fonction du débit de la forme $\mathrm{V}=a \mathrm{Q}^{0,500,7}$. Par suite, la vitesse de dissolution peut s'écrire:

$$
\frac{d \lambda}{d t}=\frac{\mathrm{K}}{\mathrm{Q}} \mathrm{V}^{\alpha} \mathrm{L}
$$


e'est-à-dire :

$$
\frac{d \lambda}{d x}=\mathrm{K} \frac{\mathrm{V}^{\alpha-1} \mathrm{~L}}{\mathrm{Q}}=\mathrm{K}^{\prime} \mathrm{Q}^{n}
$$

avec :

$$
n=(0,5 \text { à } 0,7)(\alpha-1)+(0 \text { à } 0,25)-1
$$

La valeur $\alpha=1$ (dont nous verrons plus loin

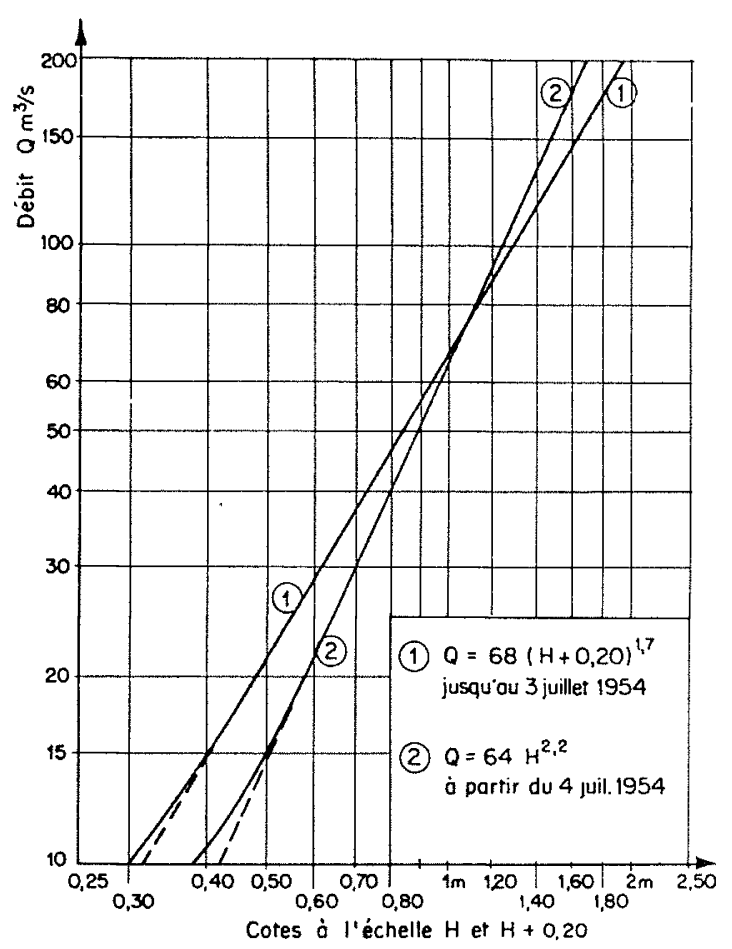

Fig. 3. - Gave de Pau. Station de Geu-Pibeste. Courbes de tarage. qu'elle est assez plausible) conduirait pour $n$ à des valeurs comprises entre $-0,75$ et -1 .

On voit que l'on peut retrouver ainsi un exposant différent de celui pour lequel nous avons effectué précédemment une vérification.

A l'extrême, nous avons essayé d'ajuster les mêmes points avec une loi $\Phi(Q)=1 / \sqrt{Q}$ et avons constaté qu'il est également possible d'obtenir un excellent résultat. Ceci montre, en particulier, que l'on ne peut pas compter sur la plus ou moins' grande facilité avec laquelle les points s'aligneront, pour déterminer l'exposant de $Q$ dans la formule.

c) Nous avons essayé, d'autre part, de déterminer l'influence de la vitesse de l'eau sur la vitesse de dissolution, en expérimentant au laboratoire avec des roches de nature et de granulométrie connues.

Les roches, concassées et soigneusement lavées, étaient disposées dans une colonne en verre de $60 \mathrm{~mm}$ de diamètre, et baignées par de l'eau distillée qui, amenée à la partie supérieure, s'écoulait lentement à la base de la colonne.

Dans nos expériences, la vitesse de filtration - définie comme le quotient du débit par la section de la colonne - a varié entre 3,5 et $48 \mathrm{~cm}$ par heure.

Une première série d'expériences a été effectuée le 5 mars 1954 avec du calcschiste de granulométrie 3,15 à $12,5 \mathrm{~mm}$, empilé sur une hauteur de $36 \mathrm{~cm}$. La vitesse de filtration a varié entre 11,9 et $48 \mathrm{~cm} / \mathrm{s}$; l'une des mesures a été effectuée avec de l'eau portée à la température de $35^{\circ} \mathrm{C}$. On observe une variation exponentielle de la conductivité vers une valeur asymptotique,

TABLEAU I. - ESSAI DE DISSOLUTION DE CALCSCHISTE (5 mars 1954)

INFLUENCE DE LA VITESSE DE FILTRATION

Calcschiste de granulométrie 3,15 à $12,5 \mathrm{~mm}$ disposé sur une hauteur de $36 \mathrm{~cm}$ dans une colonne de $\varnothing 60 \mathrm{~mm}$

\begin{tabular}{|c|c|c|c|c|c|}
\hline $\mathrm{N}^{\circ}$ & Débit moyen & $\begin{array}{l}\text { Vitesse } \\
\text { de filtration }\end{array}$ & $\begin{array}{l}\text { Conductivité } \\
\text { finale }\end{array}$ & $\begin{array}{l}\text { Variation } \\
\text { de } \\
\text { conductivité }\end{array}$ & Température \\
\hline Symboles & $Q$ & $\mathrm{~V}$ & $\lambda$ & $\Delta \lambda$ & 0 \\
\hline Unités & $\mathrm{cm}^{3} /$ heure. & $\mathrm{cm} /$ heure & micromhos $\times \mathrm{cm}^{2}$ & micromhos $\% \mathrm{~cm}^{2}$ & ${ }^{\circ}$ Centésimaux \\
\hline 1 & 351 & 11,9 & 31 & 25,7 & \\
\hline 2 & 500 & 17,5 & 31 & 25,7 & ambiante \\
\hline 3 & 1.375 & 48 & 28 & 22,7 & \\
\hline 4 & 473 & 16,5 & 30 & 24,7 & 35 \\
\hline
\end{tabular}

Eau distillée..... $\left\{\begin{array}{l}\rho=188,160 \omega / \mathrm{cm} / \mathrm{cm} \\ \lambda=5,3 \mathrm{micromhos} . \mathrm{cm} . \mathrm{cm}\end{array}\right.$ 
variation correspondant à la mise en régime de l'expérience. A l'ćquilibre, c'est-à-dire théoriquement au bout d'un temps infini, la conductivité de l'eau a augmenté d'une quantité qui dépend de la vitesse de filtration, comme l'indiquent le tableau I et la figure 4 . On constate que cette variation est très faible, et à peu près inversement proportionnelle à la puissance $1 / 9$ ou $1 / 10$ de la vitesse; la quantité de matières dissoutes serait donc à peu près proportionnelle au débit, tout au moins en régime de filtration. Cela véri-

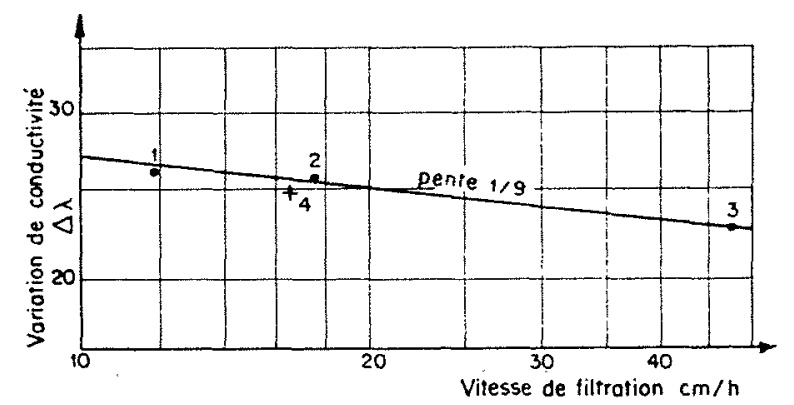

Fig. 4. - Influence de la vitesse de filtration.

5 mars 1954. Calcschiste $\Phi 3,15$ à $12,5 \mathrm{~mm}$.

fierait assez bien l'hypothèse que nous avons faite plus haut au paragraphe $a$ ).

On notera que l'influence de la température semble négligeable, tout au moins entre 15 et $45^{\circ} \mathrm{C}$.

Nous avons repris cette expérience en octobre 1956, en opérant alors avec du granit de Cauterets, d'une granulométrie de 4 à $15 \mathrm{~mm}$ environ, pour des vitesses de filtration comprises entre 3,43 et $22,5 \mathrm{~cm} / \mathrm{h}$. Les résultats sont résumés par le tableau II et la figure 5 . Bien que plus dispersés, ils sont essentiellement contradictoires avec les précédents. Ici, la variation de conductivité semble inversement proportionnelle à la vitesse de filtration; autrement dit, la quan-

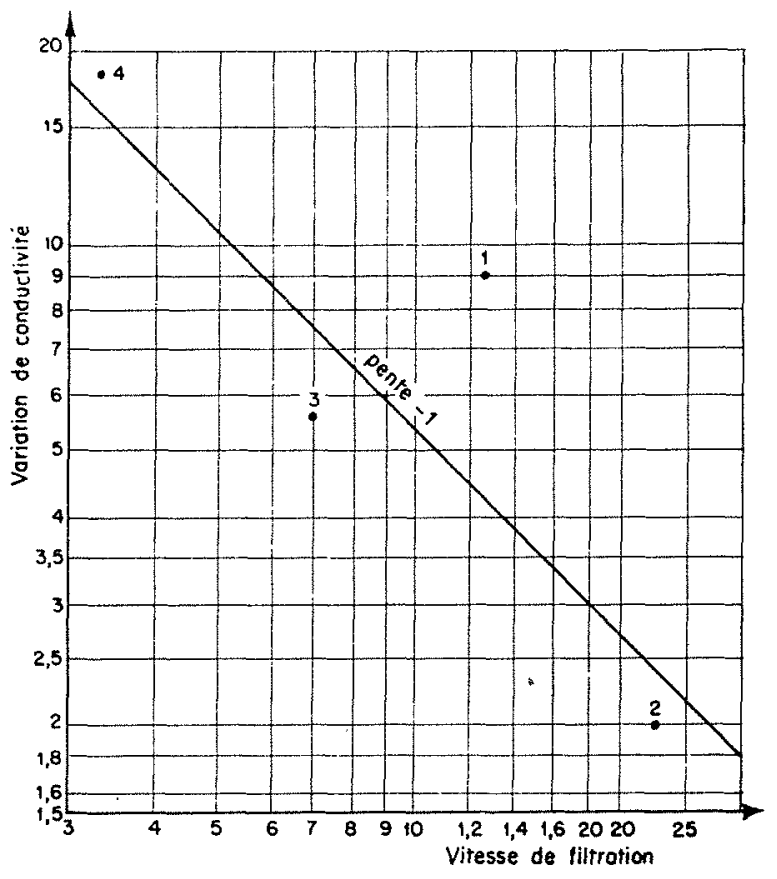

FIg. 5. - Influence de la vitesse de filtration. Octobre 1956. Granit de Cauterets. $\Phi 4$ à $15 \mathrm{~mm}$.

tité de matières dissoutes ne dépendrait pas du débit, dans le cas d'une roche pratiquement insoluble.

Peut-on admettre que la seule nature des roches puisse expliquer une telle différence? Nous nous proposons d'effectuer des expé-

TABLEAU II. - ESSAI DE DISSOLUTION DE GRANIT (Octobre 1956)

INFLUENGE DE LA VITESSE DE FILTRATION

Granit de Cauterets, de granulométrie 4 à $15 \mathrm{~mm}$, disposé sur une hauteur de $55 \mathrm{~cm}$ dans une colonne de $\varnothing 60 \mathrm{~mm}$

Eau distilléc..... $\begin{aligned} & \stackrel{o}{i}=198,400 \omega / \mathrm{cm} / \mathrm{cm} \\ & 5 \text { micromhos. } \mathrm{cm} . \mathrm{cm}\end{aligned}$

\begin{tabular}{|c|c|c|c|c|}
\hline $\mathrm{N}^{0}$ & Débit moyen & $\begin{array}{c}\text { Vitesse } \\
\text { de filtration }\end{array}$ & $\begin{array}{l}\text { Conductivité } \\
\text { finale }\end{array}$ & $\begin{array}{l}\text { Variation } \\
\text { de conductivité }\end{array}$ \\
\hline Symboles & Q & $\mathrm{V}$ & $\lambda$ & $\Delta \lambda$ \\
\hline Unités & $\mathrm{cm}^{3} /$ heure. & $\mathrm{cm} /$ heure. & . micromhos $\times \mathrm{cm}^{2}$ & micromhos $\times \mathrm{cm}^{2}$ \\
\hline 1 & 360 & 12,7 & 14 & 9 \\
\hline 2 & 640 & 22,5 & 7 & 2 \\
\hline 3 & 200 & 7,1 & 10,5 & 5,5 \\
\hline 4 & 97 & 3,43 & 23 & 18 \\
\hline
\end{tabular}


riences plus nombreuses et plus complètes sur ce phénomène.

d) Une autre solution expérimentale consiste à analyser la forme (2) de la loi de variations de conductivité, en étudiant de façon détaillée les profils en long de conductivité d'un cours d'eau pour un certain nombre de valeurs du débit Q. C'est l'objet du paragraphe suivant.

\section{APPLICATION A UN COURS D'EAU UNIFORME SANS AFFLUENT VERIFICATION DE LA LOI CONDUCTIVITE. - ABSCISSE}

L'étude de plusieurs profils en long de conductivité d'un cours d'eau convenablement choisi permettra de vérifier la loi de variation de la conductivité en fonction de l'abscisse, ainsi que de préciser l'influence du débit.

Nous avons vu que la forme (2) s'appliquait à débit constant à un cours d'eau uniforme; nous avons de plusi imposé au tronçon choisi d'être facilement accessible par la route pour effectuer les prélèvements.

Ces diverses conditions sont à peu près remplies par le gave de Pau, entre le pont de Tilhos (près d'Argelès) et le pont de Coarraze, soit sur une longueur de près de $35 \mathrm{~km}$. De part et d'autre de Lourdes, le Gave ne recoit en effet, comme le montre la figure 6 , que des affluents de très

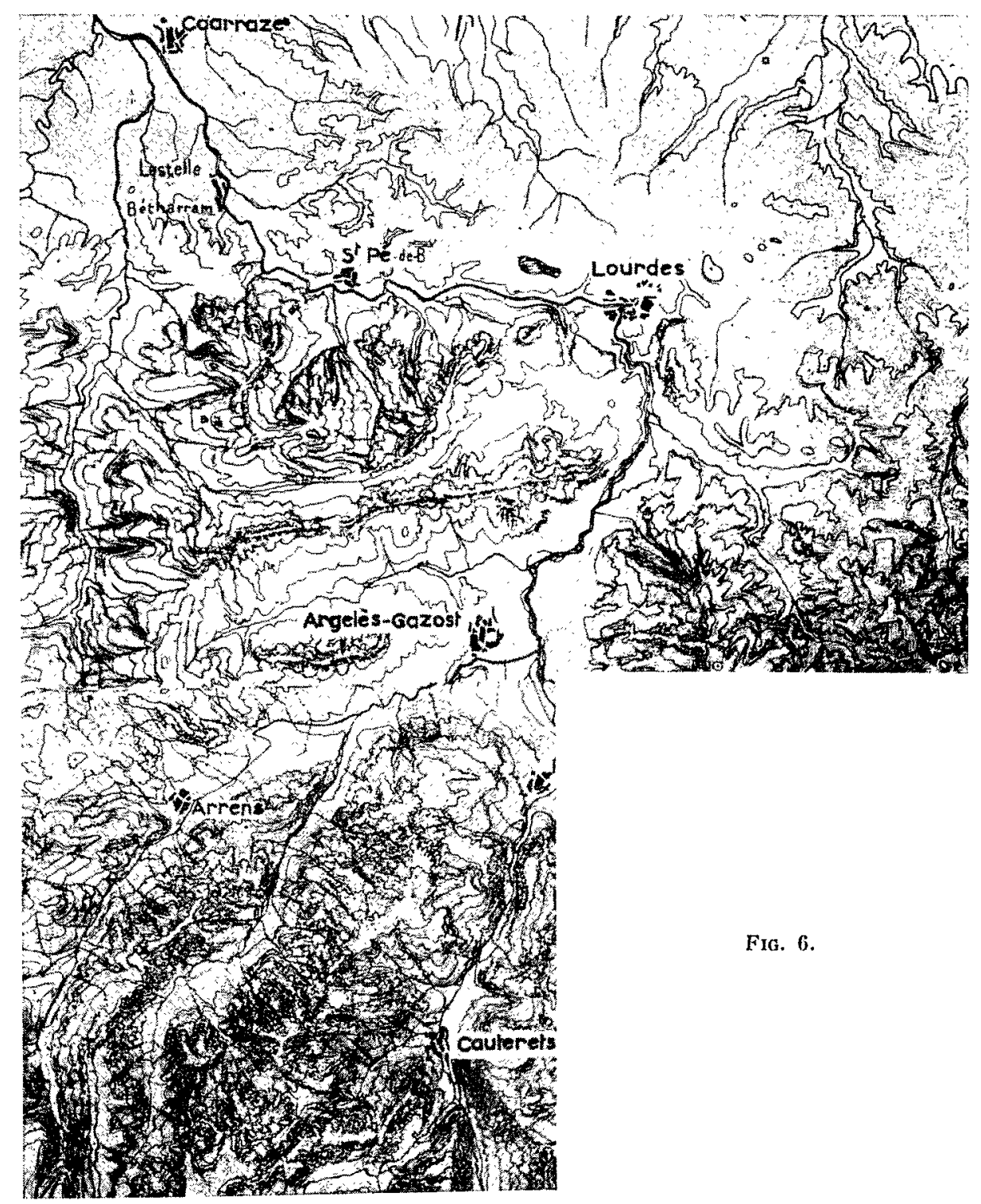


TABLEAU III - PROFILS EN LONG DES CONDUGTIVITÉS DANS LE GAVE DE PAU

\begin{tabular}{|c|c|c|c|c|c|c|c|c|c|}
\hline & $\mathrm{N}^{\circ}$ & 1 & 2 & 3 & 4 & 5 & 6 & 7 & 8 \\
\hline \multirow{3}{*}{ LIEU } & Date & $\mid 18-12-51$ & $26-12-51$ & $30-12-51$ & $31-1-52$ & $8-2-52$ & $5-2-52$ & $8-2-52$ & $12-10-56$ \\
\hline & $\overline{\text { Débit } \mathrm{m}^{3} / \mathrm{s}}$ & 17,9 & 17,9 & 30 & 19,85 & 230,6 & 107,5 & 45,4 & 28 \\
\hline & $\begin{array}{c}\text { Distances } \\
\mathrm{kmm}\end{array}$ & & & & & & & & \\
\hline $\begin{array}{l}\text { Aval pont de Tilhos. } \\
\text { Pont chemin de fer. }\end{array}$ & $\begin{array}{l}0 \\
22\end{array}$ & 161 & 158,5 & 171 & & & 164,5 & 171,2 & $\begin{array}{l}148 \\
152\end{array}$ \\
\hline Geu-Pibeste $\ldots . .$. & $\begin{array}{l}2,2 \\
6\end{array}$ & 171 & 173 & 185 & 182 & 128.0 & 168 & 179.8 & 154 \\
\hline $\begin{array}{l}\text { Pont Neuf.............. } \\
\text { Aval Pont Neuf..... }\end{array}$ & $\begin{array}{l}8,8 \\
9\end{array}$ & & & & & & & & $\begin{array}{r}147 \\
153,8 \\
159,5\end{array}$ \\
\hline $\begin{array}{c}\text { Passerelle (amont } \\
\text { Lourdes) ....... }\end{array}$ & 9,5 & 176 & 188 & 203,5 & 190,5 & 133.0 & & & 161,5 \\
\hline $\begin{array}{c}\text { Amont usine (aval } \\
\text { Lourdes) } \ldots \ldots\end{array}$ & 13,2 & & & & & & 175 & 182,9 & \\
\hline Pont de Rieulhès..... & 21,45 & & & & & & & & 171 \\
\hline $\begin{array}{l}\text { Amont Betharram. } \\
\text { Pont de Lestelle... }\end{array}$ & $\begin{array}{l}27,2 \\
29,25\end{array}$ & & & & $\begin{array}{l}206 \\
206\end{array}$ & 137,9 & & 193,6 & 170,2 \\
\hline Pont de Coarraze... & 35,35 & & & & 206,5 & & & & $\left\{\begin{array}{c}172,2 \\
173\end{array}\right.$ \\
\hline \multicolumn{2}{|c|}{ Pente relative (en $10^{-3} / \mathrm{km}$ ) } & 19 & 26 & 30 & 30,8 & $\overline{5,5}$ & 10 & $\overline{12,8}$ & $(6,7)$ \\
\hline
\end{tabular}

Les valeurs sont celles de $\lambda$ en micromhos $\times \mathrm{cm}^{2}$.

faible importance. En amont du pont de Tílhos se trouve le confluent du Gave d'Azun ou gave d'Arrens et un peu en aval du pont de Coarraze celui du gave d'Ouzom.

D'autre part, le gave de Pau coule essentiellement dans cette région sur des terrains détritiques en grande partie constitués de dépôts morainiques. Les caractéristiques hydrauliques (largeur du lit, profondeur, vitesse) sont, sinon constantes, du moins du même ordre; enfin, le gave de Pau est longé sur presque tout son parcours par des routes nationales.

Entre décembre 1951 et octobre 1956, nous avons effectué un certain nombre de profils en long de conductivité dont huit ont pu être retenus pour cette étude; plusieurs ont dù être éliminés, car ils correspondaient à des périodes où le débit, ou la variation des caractéristiques de l'eau en amont, expliquaient les anomalies observées.

En particulier, nous avons noté une fois une baisse de conductivité très marquée et qui semblait se déplacer à la vitesse de l'eau. Nous avons pu vérifier qu'il s'agissait d'un lâcher de quelques heures effectué par l'usine d'Arrens et en provenance du lac de Migoëlou, dont les eaux sont beaucoup plus pures que celle du gave de Pau (30.000 ohms au lieu de 5 à 6.000).

Les résultats de ces mesures sont rassemblés dans le tableau III. Une assez grande dispersion de ces résultats est due à l'insuffisante validité de nos hypothèses et à d'inévitables mélanges avec quelques sources plus pures ou, au contraire, avec des eaux polluées par les riverains.

Le tracé des profils en long n'a été possible qu'en admettant la même valeur de $\lambda_{m}$ pour tous ces profils. Cette hypothèse n'est pas parfaitement justifiée car elle revient à admettre que la saturation correspondant à l'écoulement dans le tronçon intéressé (et théoriquement obtenue à une distance infinie) n'est pas influencée par la composition des eaux arrivant au pont de Ti-

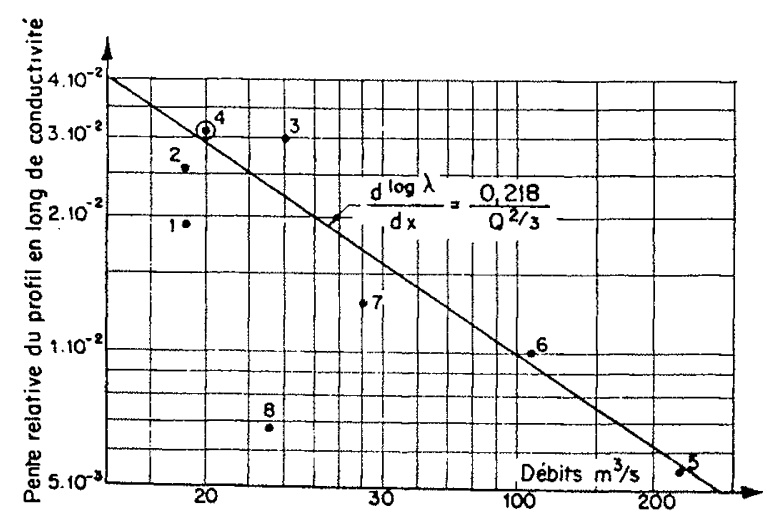

Fic. 7. - Gradient de conductivité relative en fonction du débit. 
lhos, ce que nous n'avons pas' encore eu l'occasion de vérifier.

Sous la forme (2) que nous avons donnée plus haut, les profils en long de conductivité sont représentés par des droites dont la pente, ou gradient de conductivité relative, nous donne cha-

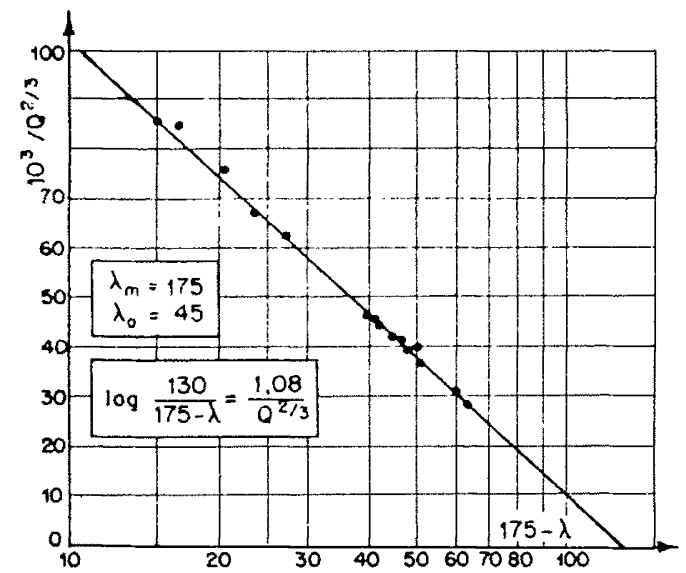

Fig. 8. - Gave de Pau. Geu-Pibeste. Crue de mars 1951. Variation de la conductivité en fonction du débit. que fois une valeur de la fonction $\Phi(Q)$ applicable au cours d'eau étudié.

La figure 7 donne les variations de ce gradient en fonction du débit.

On constate une grande dispersion des points expérimentauxi dont certains ont, toutefois, à priori, une valeur contestable. On peut en déduire pour $\Phi(Q)$ une expression de la forme $\mathrm{K} / \mathrm{Q}^{n}$, l'exposant $n$ n'est pas significativement différent de $2 / 3$.

Nous pouvons donc, pour l'instant, reprendre la forme (2) exprimant les variations de $\lambda$ en fonction de l'abscisse et du débit et la mettre sous la forme (4):

$$
\log \frac{\lambda_{m}-\lambda_{0}}{\lambda_{m}-\lambda}=\mathrm{K}\left(x-x_{0}\right) \cdot \mathrm{Q}^{-2 / 3}
$$

Avec cette nouvelle forme, les résultats présentés figure 2 donnent la figure 8 , où l'on constate que la valeur de $\lambda_{m}$, correspondant à la saturation, est différente de celle obtenue avec la première loi examinée.

\section{REMARQUE SUR LES VALEURS OBTENUES POUR LA SATURATION FAUSSE SATURATION - VERIFICATION EXPÉRIMENTALE}

On peut trouver anormal, de considérer comme saturées des eaux dont la résistivité est encore de l'ordre de 4.000 à $6.000 \mathrm{ohms} / \mathrm{cm}$, en particulier lorsque ces eaux contiennent une faible quantité de chlorure de sodium. Ce sel est, en effet, très soluble et, de plus, son ionisation conduit à augmenter fortement la conductivité de l'eau. N'ous avons, observé à $18^{\circ}$, sur de l'eau saturée de sel gemme de Dax, une résistivité voisine de 8 ohms $/ \mathrm{cm}$. Il en est d'ailleurs ainsi, quoique à un degré moindre, pour un certain nombre d'autres sels que l'on rencontre dans les eaux naturelles.

En réalité, nons pensons qu'il s'agit là d'une fausse saturation qui n'est pas contradictoire avec nos hypothèses, et peut s'expliquer de deux manières :

a) Les eaux coulent sur un bassin exempt de chlorure de sodium, sauf dans une toute petite région où un ruisseau deviendra particulièrement salin; si les débits d'un tel petit affluent et de l'ensemble du bassin versant restent proportionnels (ce qui est souvent vrai pendant toute une période de l'année hydrologique), il faudra toujours une grande quantité d'eau pour avoir une quantité assez limitée de chlorure de sodium (ou de tout autre sel); la concentration maximum obtenue est dif- férente de la saturation réelle, mais entre dans nos calculs exactement de la même façon;

b) Nous avons pensé également que les roches, même les plus réfractaires à la dissolution par l'eau, pouvaient contenir en petite quantité un sel très soluble, mais sous une forme enrobée, c'est-à-dire que ce sel serait protégé par des corps beaucoup moins solubles et beaucoup moins actifs sur la conductivité de l'eau.

Cette hypothèse, à priori particulièrement contestable, semble cependant confirmée par nos expériences. Elle expliquerait les premiers échecs que nous avons subis en essayant de reconstituer au laboratoire le phénomène de dissolution.

Si l'on opère avec de l'eau distillée et des roches prises à l'état naturel, par exemple des galets prélevés dans un lit torrentiel, on obtient une certaine valeur de la conductivité.

Lorsque l'on a pris soin de concasser le matériau pour lui donner une granulométrie à peu près uniforme, la conductivité obtenue est toujours beaucoup plus forte. Cette augmentation de la conductivité disparaît partiellement ou totalement si on lave soigneusement les pierres concassées, d'abord à grande eau, puis avec de 
l'eau distillée. Une preuve plus complète de notre hypothèse doit être fournie par les analyses chimiques détaillées des eaux contenues dans les torrents considérés et de celles saturées au laboratoire, en considérant tout particulièrement le dosage en anhydride carbonique qui a vraisemblablement une influence sur la solubilité et sur la conductivité elle-même; l'agressivité des eaux étant fonction de leur teneur en anhydride carbonique libre et semi-combiné.

\section{EXTRAPOLATION DE LA LOI DE CONDUCTIVITE - ABSCISSE LOCALISATION DES SOURCES. - APPLICATION}

La loi de variation de conductivité en fonction de l'abscisse peut être extrapolée en dehors du domaine où elle a été établie; on peut chercher ainsi à quelle distance on trouverait une conductivité soit nulle, soit au plus égale à celle des eaux de pluie ou de source. La distance ainsi obtenue ne doit pas, à priori, être égale à celle de la source du cours d'eau, mais représenter une distance équivalente à celle de cette source, correspondant au cas où le cours d'eau resterait uniforme depuis celle-ci jusqu'au tronçon de mesure.

Il se trouve que, par une heureuse compensation de variations des différents paramètres (le débit diminue vers la source, mais la surface de contact de chaque affluent diminue également, les facteurs géologiques se diversifient souvent, mais peuvent se compenser statistiquement), les distances que nous avons obtenues sont comparables à celles des principales sources du bassin versant.

Dans le cas du gave de Pau, les différentes estimations que nous avons pu faire d'après les études des profils en long conduisent à placer la source équivalente à une distance comprise entre 45 et $60 \mathrm{~km}$ en amont du pont de Tilhos. Or, précisément, les sources du gave de Pau et de ses principaux affluents (gave d'Arrens, gave d'Estaing, Marcadou, gave de Gaube, gave de Dutour, gave de Gavarnie, Bolou, Bastan) se trouvent à peu près toutes situées à des distances comprises entre 40 et $60 \mathrm{~km}$. Ceci peut n'être qu'une coïncidence qu'il ne faudrait pas être tenté de généraliser avant vérification sur d'autres bassins versants. Nous avons cependant essayé d'appliquer cette extrapolation à un cas particulièrement intéressant : celui de la rivière
Saint-Vincent. On sait que cette rivière passe au fond du gouffre de la Pierre-Saint-Martin, traverse la salle de la Verna, puis passe quatre ou cinq kilomètres plus loin au fond du Trou du Renard, qui fut exploré par M. Ravier en 1952, et débouche ensuite dans la grotte de Bentia.

Nous disposions des valeurs de la conductivité de trois échantillons seulement prélevés à la même date en trois points de cette rivière, e'està-dire dans la salle de la Verna, au trou du Renard et à la grotte de Bentia.

Malheureusement, ces trois valeurs sont incompatibles avec la forme de la loi que nous avons établie en fonction de l'abscisse, et ceci parce que les hypothèses que nous avons dû formuler ne sont' pas vérifiées : permanence dans l'écoulement incertaine; importantes réserves d'eau entre la salle de la Verna et le trou du Renard (conduisant à un déphasage de trois semaines pour le passage d'une injection de fluorescéine); non uniformité de la nature géologique des roches traversées par la rivière SaintVincent le long de son cours (en particulier existence entre le trou du Renard et la grotte de Bentia de bouchons détritiques qui, d'une part, ralentissent l'écoulement, et, d'autre part, lui offrent une plus grande surface de contact et, par suite, peuvent conduire à une valeur différente de la saturation).

Bien que cette tentative se soit soldée par un échec, la méthode pourrait cependant se montrer fructueuse dans d'autres cas; il serait cependant nécessaire de vérifier qu'elle s'applique à peu près correctement sur un certain nombre de bassins versants géologiquement homogènes ou hétérogènes.

\section{CONCLUSION}

L'étude que nous venons de présenter est loin d'être terminée; elle s'appuie souvent sur des hypothèses insuffisamment vérifiées; d'autre part, plusieurs points importants n'ont pu en- core être abordés. Aussi espérons-nous pouvoir poursuivre les travaux entrepris et conduits jusqu'ici de façon intermittente.

Des expériences déjà en cours nous fourni- 
ront des indications sur la répartition des conductivités dans une section transversale d'un cours d'eau. Nous espérons mettre ainsi en évidence l'existence d'un faible gradient transversal de conductivité montrant l'influence du fond et des berges.

Nous comptons d'autre part faire effectuer des analyses détaillées des eaux prélevées et rapprocher la conductivité des eaux des teneurs en chacun des différents sels dissous. De plus, nous ignorons à peu près tout des influences réciproques de sels afférents sur leurs saturations respectives.

A cause de ces lacunes, nous n'avons pas voulu exploiter les formules mathématiques éta- blies pour les variations de conductivité. D'ailleurs, ces formules, bien que de forme simple, conduisent à des calculs compliqués lorsqu'on veut les appliquer à un bassin versant de structure même peu complexe. Déjà leur application au cas d'un confluent, même en terrain homogène, est peu abordable si l'on n'impose pas aux débits de rester proportionnels.

On peut alors penser que les relations conductivité-débit à une station ne sont suivies sans trop d'écarts que lorsqu'une compensation statistique vient heureusement masquer les causes d'une dispersion qui, sans cela, serait énorme. Dans ce sens aussi, nos recherches pourront peut-être rendre plus sûre l'exploitation des mesures de résistivité.

\section{DISCUSSION}

(Président : M. Koch)

M. le Président félicite MM. Bonnis et Ravien pour leur travail et relève surtout l'honnêteté scientilique avec laquelle ils l'exposent. Ils font ainsi preuve d'un souci d'objectivité auquel il tient à rendre hommage. Il suggère qu'à l'occasion du Congrès de Toronto fGéophysique et Géologie), il serait intéressant de confronter ces recherches avec celles qui ont pu être poursuivies à l'étranger.

M. Archambault, pour sa part, lient d'abord à souligner l'intérêt execptionnel en tant qu'instrument d'étude des eaux superficielles ou souterraines, du résistivimètre mis au point par les Services d'E.D.F. et utilisé par MM. Bonnin et Ravien. Dans l'exemple d'eaux d'une résistivité moyenne de $5000 \mathrm{ohms}$, avec un résidu sec de l'ordre de $100 \mathrm{mg}$, l'analyse chimique peut doser au mieux le milligramme par litre, tandis que la résistivité, déterminée par un appareil à $1.10^{-4}$ ohms près, permet une investigation 100 fois plus précise. Toutefois, observant que la résistivité de l'eau au moment du prélèvement peut s'écarter sensiblement de celle mesuréc au laboratoire un certain temps après, il formule le désir que soient mis au point des apparcils susceptibles de permettre des analyses sur place, fùt-ce avec une précision un peu moindre.

Il remarque, d'autre part que si le troncon du Gave de Pau étudié sur une longueur de $35 \mathrm{~km}$ ne comporte pas d’affluents importants, il bénéficie sans aucun doute, et pour cette raison même d'ailleurs, d'apports occultes provenant du déversement des nappes souterraines de bordure.

Qu'il s'agisse des alluvions anciennes du Gave ou surtout des calcaires et marnocalcaires, crétacés et juras siques, qui forment le substratum de la valléc à l'aval d'Argeles, les formations encaissantes doivent véhiculer un débit global important : ces nappes latérales sont d'ailleurs beaucoup plus minéralisées (plusicur's centaines de mg, correspondant à une résistivité de quelques centaines d'ohms) que l'eau du Gave, dont le bassin versant au-dessus d'Argelès est essentiellement grani- tique et primaire. L'ordre de grandeur de ces apports souterains et leur minéralisation moyenne ne pourraicnt être déterminés que par une étude de terrain. Mais on peut affirmer sans crainte d'erreur que c'est à eux et, pour une part moindre, aux déversements (cités daus le rapport) d'eaux usées par les agglomérations, qu'est dû l'accroissement de minéralisation relevé de l'amont vers l'aval, le délavage des alluvions du lit. mineur ne jouant probablement qu'un rôle négligeable.

M. le Président pense que d'une manière générale l'un des intérêts les plus grands de la méthode de conductivité tient précisément au fail qu'elle peut faire intervenir, non seulement les éléments superficiels comme cenx des jaugeages dans un cours d'enu, mais aussi les apports des nappes souterraines ou d'allluents plus ou moins souterrains.

M. Ravier tient a préciser qu'une des raisons qui ont fait choisir pour les mesures le bief amont entre Argelès et Saint-Pé du Gave de Pau est que ce bief a encore une pente telle que les influences de la nappe phreatique $y$ sont pen sensibles, tandis qu'i l'aval les apports de cette nappe domaient des variations de conductivité dans un sens ou dans l'autre.

M. le Président reconnait qu'il est normal dans l'étude des procédés de ces recherches, minutieuse et difficile, de choisir des régions où le facteur souterrain joue peu; mais on peut se demander si, dans l'application, un des intérêts pratiques de la méthode ne sera pas, au contraire, de dégager l'influence des apports souterrains ou des pertes dans le sous-sol.

M. Bonnin pense qu'en effet cetle méthode peut avoir, entre autres applications, celle de délecter d'une façon très simple les fuites d'une galeric sans interrompre son fonctionnement, par exemple en injectant pendant un temps relativement limité une solution assez concentrée de ClNa, et en suivant les variations de la résistivité des sources proches de la galerie, celle-ci étant parcourue par un débit convenable pendant l'essai. 